\title{
Mode I and II Wear in Ceramic on Ceramic Total Hip Arthroplasty: A Case Report
}

\author{
Kunal Mohan ${ }^{1}$, Niall P. McGoldrick ${ }^{1}$, Conor O’Driscoll ${ }^{1}$, John F. Quinlan ${ }^{1}$
}

\section{Learning Point of the Article:}

Wear between ceramic implants in THA is infrequently reported, and as such awareness of this uncommon phenomena as well as its potential causative factors is important when considering either primary or revision THA involving ceramic bearing components.

\section{Abstract}

Introduction: Elective total hip arthroplasty (THA) is a common procedure and has been associated with favorable outcomes in both elderly and younger patients. A variety of implant and articular components exist so to allow surgeons to make a patient-specific choice with the intent of maximizing both patient outcomes and implant survival.

The utilization of ceramic bearing surfaces during THA has become increasingly prevalent, with their tribological profile conveying favorable wear and osteolysis-resistant properties. Typically, ceramic articulating components are most susceptible to failure through brittle fracture or complications such as squeaking.

Case Report: This case describes a 68-year-old Caucasian male who underwent revision of ceramic on ceramic THA due to pain, with intraoperative analysis revealing evidence of gross Mode I acetabular ceramic component wear with a resultant Mode II wear articulation between the ceramic femoral head and acetabular liner.

Conclusion: Wear between ceramic implants in THA is infrequently reported, and as such awareness of this uncommon phenomena as well as its potential causative factors is important when considering either primary or revision THA involving ceramic bearing components.

Keywords: Hip, arthroplasty, revision, ceramic, wear.

\section{Introduction}

Total hip arthroplasty (THA) for the treatment of osteoarthritis is a common surgical procedure [1]. Advances in implant technology and surgical techniques have allowed for significant benefit in regards pain relief and function to be conferred to both elderly and younger patients [2].

An important consideration during THA is to utilize a durable implant [2], with an expected THA life expectancy of 20 years in about $78.9 \%$ of patients [3]. Success following THA is exceedingly patient-specific [3], and there are numerous options available in the orthopedic armamentarium to help optimize outcomes, particularly with regards to implant and articular component selection [4].

As the number of younger, more active patients undergoing
THA increases, the issue of implant longevity has become of paramount importance due to the risks of surface wear, osteolysis, and loosening predisposing these patients to earlier revision procedures [5]. With this in mind, the utilization of ceramic articular bearings has become increasingly prevalent due to their favorable biomaterial properties [5]. Ceramic bearings display a high Young's Modulus and are both biocompatible and bioinert [6]. Furthermore, modern articulating ceramics are both scratch-resistant and wettable and thus are thought to have extremely amenable wear and osteolytic-resistant characteristics, with the survival of up to $84.4 \%$ at 21 years described [6].

Despite these features, ceramic bearings remain at risk of failure, having traditionally been susceptible to brittle fracture due to

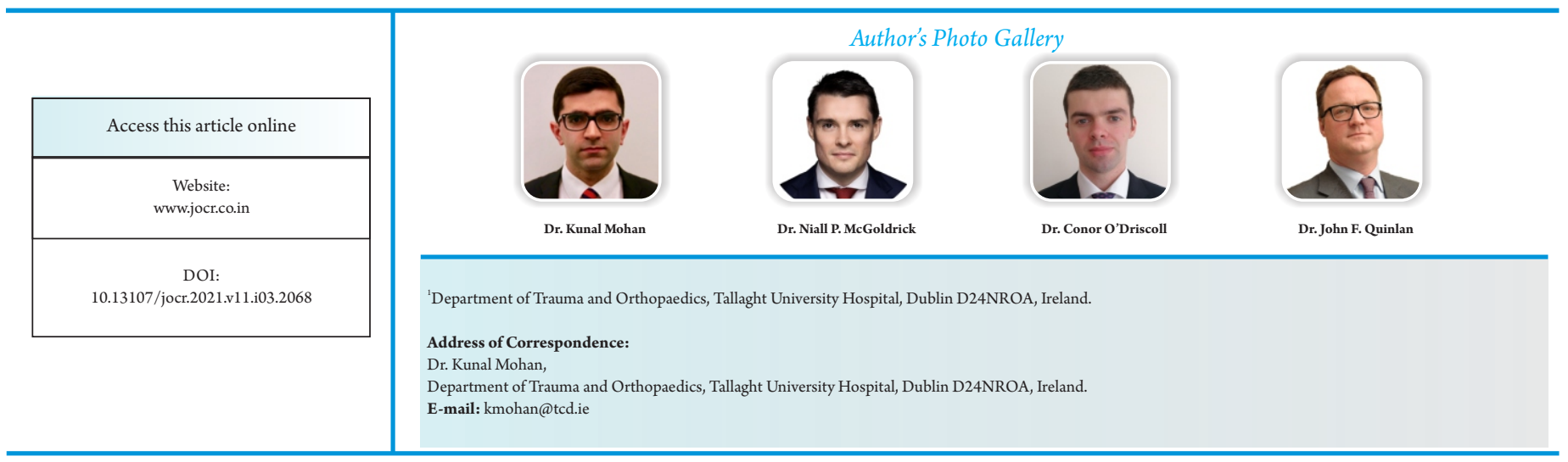

Journal of Orthopaedic Case Reports | pISSN 2250-0685 | eISSN 2321-3817 | Available on www.jocr.co.in | doi:10.13107/jocr.2021.v11.i03.2068 This is an Open Access article distributed under the terms of the Creative Commons Attribution Non-Commercial License (http://creativecommons.org/licenses/by-nc/3.0) which permits unrestricted non-commercial use, distribution, and reproduction in any medium, provided the original work is properly cited. 


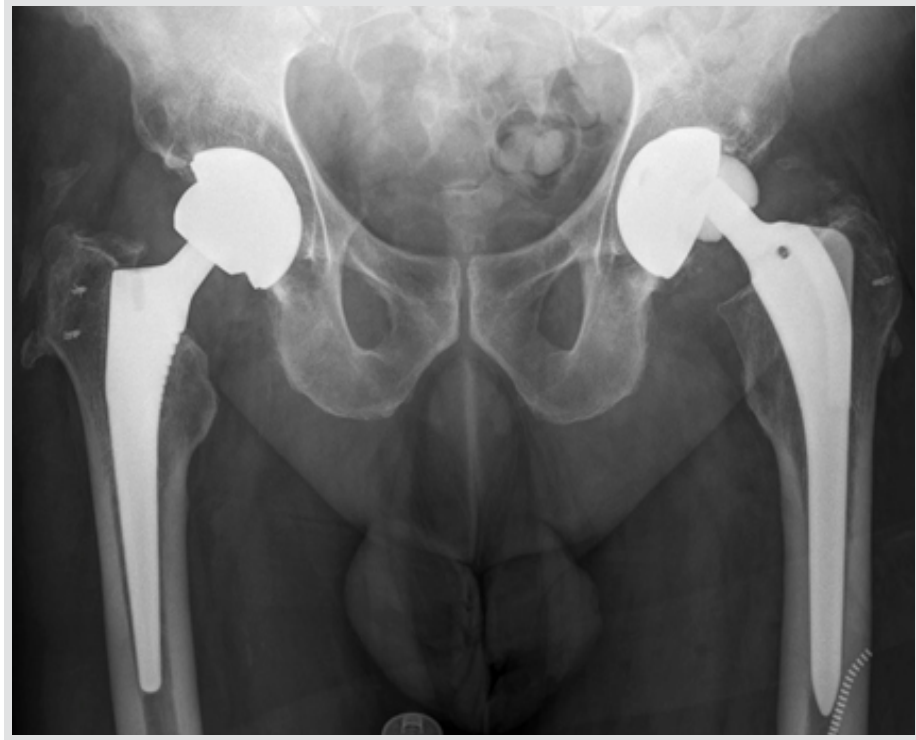

Figure 1: Pre-operative AP plain pelvic radiograph showing superior migration of femoral head of primary left total hip arthroplasty.

their high notch sensitivity [6], particularly in early generation ceramics [5]. While the risk of this substantially reduced in modern ceramic bearings [2], component failure through this method, as well as complications such as squeaking, continues to exist [6].

\section{Case Report}

A 68-year-old Caucasian gentleman presented to the Emergency Department with a 3-week history of left hip pain, clicking, and difficulty weight bearing, with no antecedent trauma. This occurred on a background history of an ipsilateral ceramic on ceramic $(\mathrm{CoC})$ uncemented THA implanted 10 years previously, with a complicated immediate post-operative course involving a dislocation requiring closed reduction. $\mathrm{He}$ suffered from no further complications and was asymptomatic up to this presentation. His background history was significant for a right uncemented CoC THA 6 years previously, type-2 diabetes, and chronic venous disease.

On examination, the patient displayed an antalgic gait with no obvious leg length discrepancy. Inspection revealed no evidence of infection at the previous THA incision. The patient suffered from pain on both active and passive movement at the hip, with an audible clicking sound throughout. There was no associated neurovascular deficit.

Plain radiographic evaluation revealed superolateral subluxation of the ceramic femoral head in relation to the ceramic acetabular component with no associated periprosthetic fracture or dislocation (Fig. 1). Serological evaluation revealed normal infectious markers, consistent with clinical assessment showing no evidence of infection. Further imaging using computerized tomography revealed

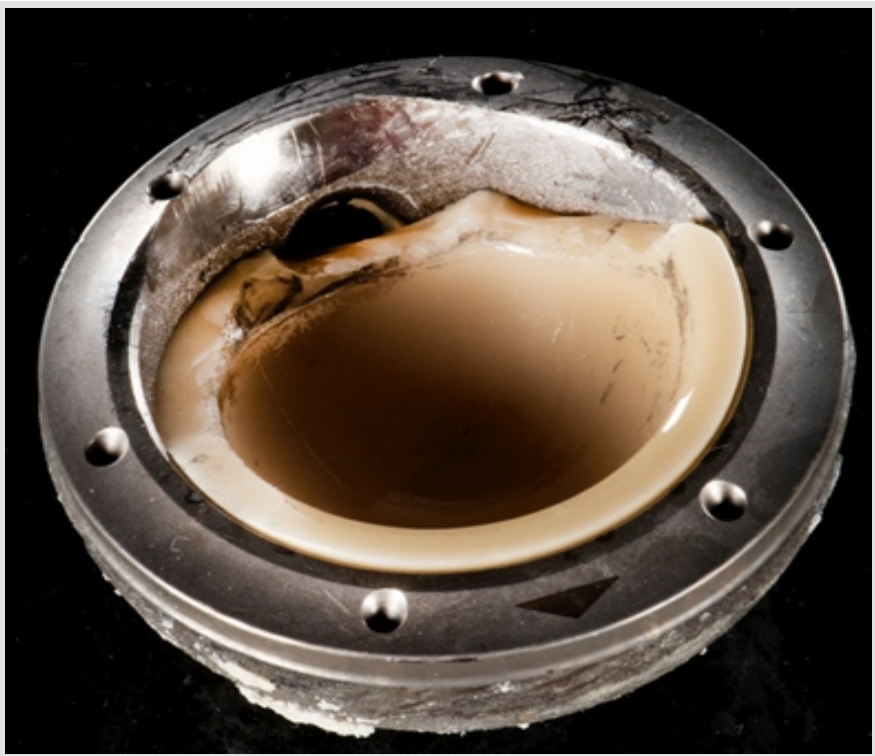

Figure 2: Clinical photograph showing wear of superolateral aspect of ceramic acetabular component.

superolateral deficiency of the acetabular component, with a diagnosis of ceramic acetabular component fracture presumed to be the mechanism behind the patient's symptoms.

Following initial inpatient management, the patient's symptoms improved, but nevertheless, he continued to suffer from discomfort and limitation of his activities of daily living (ADL's). Following outpatient re-evaluation by a specialist revision arthroplasty orthopedic surgeon, a shared decision was made to proceed to revision THA, with the intended goal to address the presumed ceramic acetabular component fracture.

This was undertaken utilizing the anterolateral incision from the index THA procedure. Inspection during exposure of the hip joint revealed no obvious macroscopic evidence of infection, osteolysis, or destruction of surrounding articular tissue. Tissue samples were taken using an aseptic technique and sent for culture and sensitivity; these identified no underlying microscopic infective cause for the patient's symptoms.

Assessment of the implanted femoral components was unremarkable, revealing a well-fixed proximally coated uncemented femoral component with no evidence of loosening or osteolysis and an intact articulating ceramic femoral head.

Evaluation of the acetabular component was, however, more significant, revealing evidence of gross wear of the superior ceramic acetabular liner, with a resultant Mode II wear articulation of the ceramic head with the underlying titanium acetabular shell (Fig. 2). There was no evidence of associated ceramic component particulate debris or fracture as had been suspected preoperatively.

The native acetabular liner and shell were thus removed and revised to a titanium alloy externally-coated acetabular shell and 


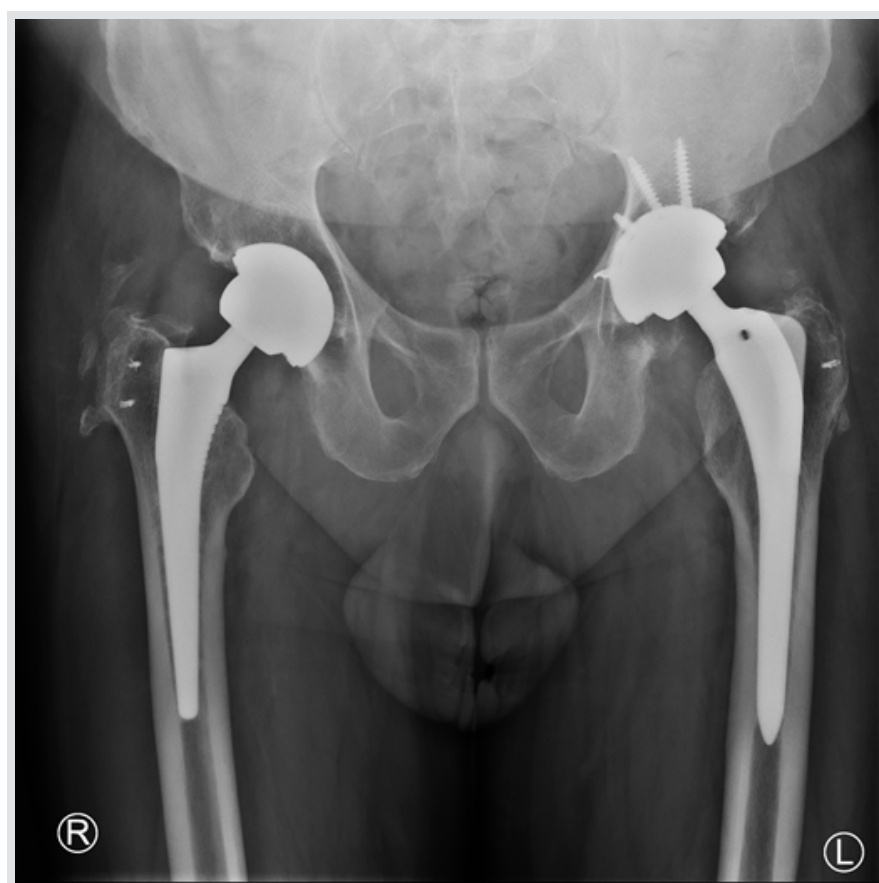

Figure 3: Post-operative AP plain pelvic radiograph showing revision of acetabular shell, cerami acetabularliner, and ceramic femoral head.

corresponding fourth-generation alumina-ceramic composite liner. The native femoral stem was left in situ as it was well fixed, with the ceramic femoral head being replaced by a corresponding fourth-generation ceramic head. The postoperative radiograph is illustrated in Fig. 3.

Post-operatively, the patient was treated with both antibiotic and thromboprophylaxis and allowed to fully- weight bear. At the 9-month post-operative interval, the patient has had a successful outcome, with complete resolution of his preoperative symptoms as well as a return to all routine ADL's.

\section{Discussion}

Articular surface wear is a major limiting factor to THA survival [7] and thus is one of the most challenging issues pertaining to implant technology [8]. This initially came to prominence following the development of Charnley Low Friction Arthroplasty, which had multiple design characteristics to help minimize wear [8]. In the following years, metal on polyethylene (MoP) became the most widespread articular surfaces utilized in THA, owing to their relatively successful outcomes [8]. The biomaterial susceptibility of both metal and polyethylene to wear and osteolysis, however, limited their survival, and as such, precipitated the development of alternative articulating surfaces [8]. Ceramic bearings were thus developed, with their theoretically favorable wear and osteolytic properties when compared to $\mathrm{MoP}$ resulting in relatively high survival rates $[5,6]$, thus making them a viable articular option during THA [8].

The common mechanisms of ceramic articular component failure are well described $[5,6,8]$, with revision surgery typically occurring due to complications such as brittle fracture, squeaking, grinding, or clunking [6]. To the best of our knowledge, ceramic failure due to a purely mechanical wear process, as described in this case, is seldom reported. However, this particular case showed evidence of apparent Mode I wear, defined as wear occurring between two intended bearing surfaces [9], of the ceramic acetabular component due to its articulation with the ceramic femoral head. This, in turn, resulted in Mode II wear, defined as wear occurring between an intended and non-intended bearing surface [9], precipitating as the ceramic femoral head articulating with the titanium acetabular shell. Indeed, these mechanisms of wears are far more frequently encountered in $\mathrm{MoP}$ articulations, with the associated hard on soft articulation causing the polyethylene to be susceptible to degradation through adhesive or abrasive processes, eventually resulting in wear and metal head articulation with the acetabular shell [8].

A number of significant observations arise from this relatively rare but clinically important finding. First, while ceramic bearings are thought to be less susceptible to wear processes than metal or polyethylene components, it is important to emphasize that this does exist, with between 0.78 and $1.4 \%$ of CoC THA articulations reported to show evidence of wearrelated osteolysis [5]. Indeed, it has been postulated that while wear debris can be created between CoC bearings, this occurs in low amounts and thus does not tend to trigger the more pronounced osteolytic reaction seen in MoP articulations [5].

The utilization of a third-generation ceramic articulation in the index THA surgery, in this case, may also be a factor behind the encountered wear process. To date, there have been four generations of ceramic components, with earlier generations at higher susceptibility of wear and failure [5]. Modern-day fourth-generation ceramic components typically consist of a composite of ceramics so to maximize their wear resistance and have been manufactured using optimized strategies so to further eliminating surface inconsistencies, with these ceramics having been shown to display wear rates of $<0.25 \mathrm{~mm} 3 /$ million cycles [6].

Outside of implant factors, a number of surgical and patient factors have been shown to precipitate an accelerated wear process in THA involving MoP articulations $[6,10]$. Extrapolation of these factors to this patient's CoC THA may offer other potential explanations behind the encountered wear. Surgical factors such as cup inclination, cup lateralization, femoral head size, and combined component version have all been suggested to affect the rate of wear in THA [10], with the appropriate component alignment of particular relevance in preventing complications in $\mathrm{CoC}$ articulations [6]. Indeed, the steep abduction angle of this patient's left acetabular component on the index AP radiograph (Fig. 1) is likely 
particular relevance in the subsequent failure of this prosthesis. This, in addition to the other factors outlined above, may have caused excessive superolateral acetabular component loading and accelerated the wear process that precipitated this patient's symptoms. In addition, the patient's relatively young age and high activity levels at the time of his index THA may have predisposed him to increase the likelihood of wear, with physical demands suggested to be a significant factor in wear rate [10].

An important consideration when revising a $\mathrm{CoC}$ articulation is stratifying the choice of the new articular components. The probability of retained ceramic fragments following $\mathrm{CoC}$ revision is significant [11]. This, in turn, can predispose the newly revised articular surfaces to potential Mode III wear, namely the articulation of two intended bearing surfaces with a third body [9]. Utilization of articular surfaces with favorable wear profiles in this scenario is thus suggested so to minimize the potential risk of accelerated wear. In practical terms, utilization of a MoP articulation for revision in those with a primary $\mathrm{CoC}$ articulation is not typically recommended, owing to the potentially significant risk of articular wear and metallosis that can result from the interaction of residual ceramic fragments with articular components more susceptible to wear $[11,12]$. Rather, revision using $\mathrm{CoC}$ bearings as demonstrated in this case is recommended in these cases due to their superior wear properties and thus improved resistance to potential Mode III wear [12].

Although this case appeared to show apparent wear between the articulating ceramic components, it is important to acknowledge other mechanisms may also precipitate implant failure. Ceramic articular components are classically susceptible to fracture [6], and indeed this characteristicled to a presumed pre-operative diagnosis of acetabular ceramic fracture being the diagnosis for this patient's symptoms. Acetabular component position has been shown to be a relevant surgical factor in both polyethylene and ceramic liner fracture $[13,14]$, and the pronounced retroversion and inclination of the native acetabular shell in this scenario may have contributed to both the early dislocation following index THA as well as a potential fracture of the acetabular shell which could precipitate wear. The dislocation itself could also have theoretically contributed to the fracture of the acetabular liner.

While fracture is a plausible cause of ceramic failure, in this scenario, pre-operative imaging showed no discernible ceramic debris and intraoperative inspection showed no macroscopic evidence of ceramic particulates that one may expect if the fracture was the underlying etiology [13]. Winston et al. have described pre-operative symptoms such as longstanding pain, crunching, and skin discoloration as well as intraoperative evidence of osteolysis, soft tissue destruction, or metallic debris in those with a delayed diagnosis of ceramic acetabular liner fracture in $\mathrm{CoC}$ articulations [15]; none of which were present in this case. Finally, the native ceramic femoral head was grossly intact and showed no evidence of having been subject to Mode III wear, which one may anticipate if fractured ceramic particulates were present for a prolonged period of time. Additional tests such as synovial fluid analysis to evaluate for microscopic ceramic debris and analysis of the explanted prosthesis can be considered to definitively outrule ceramic fracture in the revision setting [14].

\section{Conclusion}

To summarize, while ceramic bearing components utilized during THA have favorable wear characteristics and are typically associated with complications such as fracture, this case illustrates that wear is indeed a potential precipitating factor for ceramic failure and subsequent revision. Given the prevalence of ceramic components in modern-day THA, awareness of this uncommon phenomena as well as its potential causative factors is important when considering either primary

\section{Clinical Message}

Awareness of both the existence and potential etiology of the uncommon phenomena of wear between ceramic implants in THA is important when considering either primary or revision THA involving ceramic bearing components.

\section{References}

1. The Organisation for Economic Cooperaiton and Development. Health at a Glance 2017: OECD Indicators. Paris: The Organisation for Economic Cooperaiton and Development; 2017.

2. Learmonth ID, Young C, Rorabeck C. The operation of the century: Total hip replacement. Lancet 2007;370:150819.

3. Evans JT, Evans JP, Walker RW, Blom AW, Whitehouse MR,
Sayers A. How long does a hip replacement last? A systematic review and meta-analysis of case series and national registry reports with more than 15 years of followup. Lancet 2019;393:647-54.

4. National Joint Registry. The National Joint Registry 16th Annual Report 2019. London: National Joint Registry; 2019.

5. Wang T, Sun JY, Zhao XJ, Liu Y, Yin HB. Ceramic-on-ceramic 
bearings total hip arthroplasty in young patients. Arthroplasty Today 2016;2:205-9.

6. Rieker CB. Tribology of total hip arthroplasty prostheses: What an orthopaedic surgeon should know. EFORT Open Rev2016;1:52-7.

7. Karachalios T, Komnos G, Koutalos A. Total hip arthroplasty: Survival and modes of failure. EFORT Open Rev 2018;3:232-9.

8. Merola M, Affatato S. Materials for hip prostheses: A review of wear and loading considerations. Materials (Basel) 2019; 12:495.

9. Kurcz B, Lyons J, Sayeed Z, Anoushiravani AA, Iorio R. Osteolysis as it pertains to total hip arthroplasty. Orthop Clin North Am 2018;49:419-35.

10. Gallo J, Havranek V, Zapletalova J. Risk factors for accelerated polyethylene wear and osteolysis in ABG I total hip arthroplasty. Int Orthop 2010;34:19-26.
11. Gessner BD, Steck T, Woelber E, Tower SS. A systematic review of systemic cobaltism after wear or corrosion of chrome-cobalt hip implants.J Patient Saf2019; 15:97-104.

12. Im C, Lee KJ, Min BW, Bae KC, Lee SW, Sohn HJ. Revision total hip arthroplasty after ceramic bearing fractures in patients under 60-years old; mid-term results. Hip Pelvis 2018;30:156-61.

13. Traina F, De Fine M, Di Martino A, Faldini C. Fracture of ceramic bearing surfaces following total hip replacement: Asystematic review. Biomed Res Int 2013;2013:157247.

14. Tower SS, Currier JH, Currier BH, Lyford KA, Van Citters DW, Mayor MB. Rim cracking of the cross-linked longevity polyethylene acetabular liner after total hip arthroplasty. J Bone Joint Surg Am 2007;89:2212-7.

15. Winston BA, Kagan RP, Huff TW. Delayed diagnosis of catastrophic ceramic liner failure with resultant pelvic discontinuity and massive metallosis. Arthroplasty Today 2017;3:77-82.

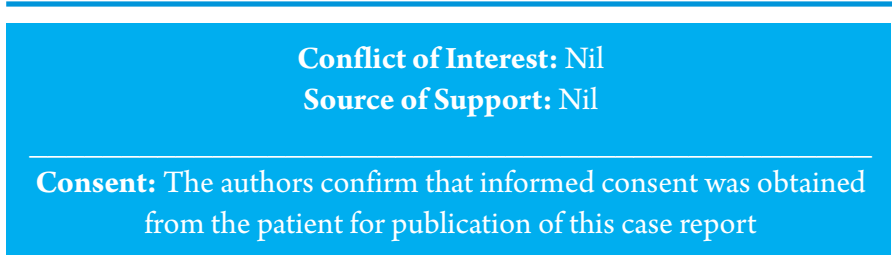

\section{How to Cite this Article}

Mohan K, McGoldrick NP, O’Driscoll C, Quinlan JF. Mode I and II Wear in Ceramic on Ceramic Total Hip Arthroplasty: A Case Report. Journal of Orthopaedic Case Reports 2021 March;11(3): 16-20. 